\title{
Risks, Benefits, Solidarity: A Framework for the Participation of Children in Genetic Biobank Research
}

\author{
Kristien Hens, PhD, Herman Nys, PhD, Jean-Jacques Cassiman, PhD, and Kris Dierickx, PhD
}

esearch on biological samples from children and pediatric biobanks can be useful for the progress of medical science. For example, genetic cohort studies can shed an important light on the interaction between genes and environment and help understand diseases such as asthma, food intolerances, autism, and attention-deficit/ hyperactivity disorder. Also, disease-specific collections can help in the understanding of the importance of gene variations in the development of the disease in question. Collections of samples and extracted DNA can typically be stored in a longer period, and research on them can change with time. Collections can be gathered primarily for research purposes, or collections originally gathered for diagnostic purposes could be re-used for genetic research.

Although useful, such collections also pose specific ethical problems that are not covered by the discussion on stored tissue samples from adults. Existing discussions often link the question whether children should and can participate to the question of minimal risk and benefit. In this paper, we explore these topics further in the context of pediatric biobanks and provide some suggestions on the ethical inclusion of children in biobank research. We shall use the term "children" rather broadly, from birth until the legal age of competence, although we admit that each phase in growing up has specific issues and therefore requires a slightly different approach. We assume that the children in question will become independent adults. The issue of mentally retarded children who will never become independent adults is an interesting topic in itself. These children will stay in the custody of parents or family members or, when deceased or otherwise unavailable, in the custody of the state when they become adults. This raises special issues about who can consent for research on samples of these children or adults. Although we do not question the rights of parents to consent for research on biological samples also in this case, the right of the state to consent to genetic research on samples from individuals in its custody is more problematic. This issue merits further reflection, but is beyond the scope of this paper.

We also admit that there is a difference between the use of collections of samples that were gathered for diagnosis and the gathering of new samples from children with the sole purpose of therapeutic research. This difference has ethical implications to which we shall come back in due course.

In the context of biobanks, some have argued that the traditional autonomy paradigm in bioethics has failed. ${ }^{1,2}$ In this respect, the principle of solidarity is used, which is put forward as an alternative to the more traditional principles that were first laid down by Beauchamp and Childress (autonomy, nonmaleficence, beneficence, and justice). ${ }^{3}$ Be- cause contributing to genetic research on stored tissue samples typically does not require too much effort from participants and because this generates potentially huge benefits for future generations, biobanks have the right to appeal to citizens to participate in such research. More specifically, this is true when the research aims to develop interventions for diseases that impair individual autonomous and social functioning. ${ }^{4-6}$

Solidarity as a principle is relatively new in bioethics. Its application to politics and society in general has been laid down by authors such as Léon Bourgeois. ${ }^{7}$ Bourgeois describes solidarity as a natural and practical middle ground between individualism and collectivism. It is natural, because it arises organically from all individuals being associated in society and this association helping individuals. In return, individuals also have an obligation toward society. Indeed, he believes that all people are born with an obligation to society. However, this is not the same as collectivism, because this obligation grows naturally from individuals only being able to thrive within society.

This way of thinking can be applied to biomedical ethics to provide an alternative to autonomy-centered principlism, without losing the necessary respect for individual values. The principle of solidarity implies that researchers in medical sciences can appeal to the solidarity of individuals, because these individuals have benefitted from earlier research and will possibly benefit from future research. However, individuals still have the right to refuse participation. Their duty consists in that they should at least consider such appeal and substantiate a refusal with valid arguments. ${ }^{8}$

Even advocates of the solidarity principle make an exception for children. ${ }^{9}$ On the one hand, until they reach the age of competence, children have limited autonomy, and so their wishes are unknown. So even when there is a strong appeal to participate, they are not in the position of considering a substantiated refusal. Also, in everyday life they are exempt from many adult duties. In this respect, the moral appeal one could make on children to participate in research may be restricted. On the other hand, some authors have argued that we must not assume, for incompetent children, that they would not want to participate in research if they had the capacity to consent. ${ }^{10}$ Moreover, we do expect certain civilized and solidary behavior from our children in everyday life. Exercising

From the Centre for Biomedical Ethics and Law (K.H., H.N., K.D.) and Department of Human Genetics (J-J. C.), Katholieke Universiteit Leuven, Leuven, Belgium

Supported by FWO Flanders (G029107). The authors declare no conflicts of interest.

0022-3476/\$ - see front matter. Copyright $\odot 2011$ Mosby Inc.

All rights reserved. 10.1016/j.jpeds.2010.12.036 
Table. Overview of the arguments

Themes

Background

Privacy risks

Risks of physical and emotional burdens

Risks of breaches of values

Benefits

Recommendations

\section{Arguments}

Researchers have the right to appeal to children and their parents to ask them to participate in biobank research. However, this right is limited by the special vulnerability of children. Issues of risks and benefits of participation should be considered

There is a risk that genetic information is abused by third parties such as insurance companies and employers. In our opinion, this does not warrant the exclusion of children from biobank.

Children may experience pain or be frightened of certain methods used to gather samples.

Genetic research on stored tissue samples may spread over longer periods. Children may have different values than their parents and may not agree with certain research to which parents consented.

Requirement of direct benefit is not applicable to pediatric biobanks.

Requirement of group benefit is difficult to substantiate in case of genetic research.

Requirement that it can only be done on children when it cannot be done on adults is a straightforward one.

Issues of privacy risks should be solved a priori on societal level (just society with legally controlled public health system) and with adequate data protection measurements on the level of biobank governance.

Research on children should not burden children physically or emotionally. Preferably, samples gathered in a diagnostics context should be used. When this is not possible, the research protocol should be first assessed by an ethics committee and then by the parents, the researcher, and the specific child.

A child's current and future values should be respected, and a child should be given the opportunity to re-consent when research on his or her samples takes a different form from what was originally intended.

Research on children's tissue should only be done when the same research cannot be done on tissue from adults. some level of solidarity during childhood may help them develop into more solidary adults. Therefore, we propose a model of limited solidarity for children. Because some research, which is potentially beneficial to future generations of children, just cannot be performed on stored tissue samples from adults (for example, if the disease develops or occurs in childhood), children can be asked to enroll for such research. But children are different from adults because it is generally assumed that they are vulnerable persons and so they should be shielded from too risky or burdensome procedures. Traditionally, discussions about children's participation in research and biobanks have focused on the belief that there should be no more than minimal risks and at least some benefit to the participating individual or the group to which he or she belongs. ${ }^{11,12}$ In the next sections, we shall discuss these principles, elaborate on which risks form a reasonable restriction to the right of biobank researchers to appeal to children to participate in genetic biobank research, and discuss the issue of benefits. We have given an overview of the arguments that are used in the rest of this text (Table).

\section{Minimal Risks}

In pediatric research, the principle is often held that children can only participate in research that offers no direct benefit to them when the risks are minimal or, in certain conditions, a minor increase greater than minimal risk. ${ }^{13}$ Also, the discussion of whether children can participate in biobank research is sometimes framed in minimal risk. Some guidelines and position papers mention "minimal risk" in the context of children. ${ }^{11,14,15}$ The Council for International Organizations of Medical Science International Ethical Guidelines for Biomedical Research Involving Human Subjects gives a definition of what minimal risk might entail: "The risk from research interventions that do not hold out the prospect of direct benefit for the individual subject should be no more likely and not greater than the risk attached to routine medical or psychological examination of such persons." 16 Other interpretations of the minimal risk standard refer to situations children encounter in everyday life. ${ }^{17}$ Wendler et al have pointed out that both the reference to everyday life and to routine medical examinations pose some problems. For example, the risks posed to children by riding in a car or going to the swimming pool are much higher than the physical risks linked to venepunctures. ${ }^{13}$ Would this mean that children could possibly be exposed to higher risks in research than they are now? The risks may be more intangible; for example, trauma or psychological damage resulting from fear may be non-existent for one child, but greater for another child. Children with a medical history have more experience with examinations than healthy children. It seems unfair, however, that this would make them preferred research subjects. The issue is further complicated when the research in question is on stored tissue samples, such as is the case in biobank research. In this case, risks will depend on the way information is gathered and the type of biobank. The reuse of samples that were gathered in a diagnostics context will pose different (and possibly less) issues than research that gathers blood samples from children specifically for research purposes Also, the genetic aspect could entail possible risks that do not occur in other types of pediatric research. Because these samples could be used for longer periods, until and after the child reaches adulthood, additional risks that are not analogous to risks encountered in one-time clinical trials may also be introduced.

Eriksson and Helgesson ${ }^{18}$ believe that there is less risk involved in biobank research than in human subject research because of the limited physical risk. However, they acknowledge there may be other risks, such as moral harms, because the storage and use of samples may violate the autonomy, privacy, or personal integrity of participants. Also, they see the possibility of non-physical harm; for example, 
information derived from samples can be used to the disadvantage of the person by employers or insurance companies. ${ }^{18}$ In the following section, we shall discuss 3 types of possible risks related to pediatric biobanks: privacy risks related to genetic information, physical and emotional harms, and disrespect of values. We shall assess each of them and see whether they warrant the exclusion of children from biobank research or, if they do not, how they can be best tackled.

\section{Privacy Risks Related to Genetic Information}

Much has been written about the risks to which the individual can be exposed when his or her genetic information is determined. As Boenink and Van der Burg have argued, in the context of genetic testing, risk discourse is predominant: the main focus lies on the reduction of the occurrence of certain genetic conditions, conditions that may occur in the future of the tested individual. ${ }^{19}$ The risks associated with genetic information available through genetic research and those associated in biobanks are different at first sight, but they are two sides of the same coin. These risks are predominantly privacy related. Genetic information, it is stated, can be misused by third parties such as insurance companies and employers if they gain access to the biobank..$^{20,21}$ These risks are also similar in the case of the reuse of diagnostic collections or the gathering of samples specifically for research. They are enhanced by such research possibly being spread over time and samples and information being stored for longer periods, and future risks or possible privacy breaches are hard to foresee on installation of the collection. In this respect, both types of risks associated with genetic information (genetic testing to prevent risks and genetic research generating risks) are related, because they are concerned with the specific nature of genetic information. Because genetic information is stable across a lifetime and contains information about potential health problems in the future, it is often considered a "future diary." ${ }^{2}$ As such, the genetic risks are good examples of what Ulrich Beck describes in his seminal work Risk Society. ${ }^{23}$ Such society is confronted with risks that stretch across borders and time and are the result of technological changes and that can no longer be contained or prevented by individual choices. But risks are often socially constructed and hard to objectify. As an extreme example, boarding a plane is experienced by many people as more risky than riding in a car, because the former is a less familiar experience than the latter. ${ }^{13}$ Genetic information may fit in this pattern, because of the relatively newness and uncanniness of the matter. The issue at stake here is whether the risk associated with genetic information is real enough to warrant the exclusion of children from biobank research, and, if not, whether restrictions should apply and which ones. To answer this, we must first consider the objective reality of this type of risk, the relation it has to other risks children face, and the possible limitations these risks would pose on pediatric research.

First, what is the objective reality of the risks associated with genetic information? These risks bear similarities to a concept called "genetic exceptionalism." Genetic exceptionalists con- sider genetic information to be fundamentally different from other medical information, because it contains information about a person's medical future and might divulge information about relatives and be used for purposes of discrimination. ${ }^{24}$ However, other authors have specified that other types of medical information can also contain such information, and it would be equally harmful if such information is misused by employers and insurers. People do not typically question health examinations offered by employers or medical data stored in files at the general practioner's office, and most people are not afraid that such information is misused by insurance companies. In a research context, how realistic is it that insurance companies would hack into databases or bribe researchers to provide information about certain individuals, especially in countries where there is an elaborate and legally controlled public health care system? We do agree that there is something special about genetic information. First, it is familial in nature and shared with other people. Second, potentially a huge amount of information can be gathered from one single source. Especially with the advent of whole genome sequencing techniques, this could eventually lead to the publication of a person's entire genome. It has recently been shown that it is possible to re-identify completely anonymous samples. ${ }^{25}$

Our second question is whether these possible risks warrant the exclusion of children from biobank research, as some have argued. ${ }^{26}$ We do not think that the privacy risks associated with pediatric biobank research are higher than other risks children encounter in everyday life. If adults release health information about themselves without reasonable fear of this information being misused by third parties, this is especially true in the case of children. One could argue that much potentially harmful medical (but non-genetic) information is stored in databases of public health services monitoring children from birth until they reach adulthood. Files may contain the length and weight of children at a certain age, whether they are breast fed or bottle fed, and their dental care, all of which may potentially affect the future health of the individual in question, on the basis of statistical assumptions. Such information is typically also used for epidemiological research without explicit consent of parents or children and may be more easily accessible to and interesting for insurance companies or employers than genetic research databases. Thus potential third-party access to data is no sufficient risk to warrant non-inclusion of children in biobanks.

Third, we must ask whether the existence of certain privacy risks may warrant certain limitations. A recent policy paper in Science has argued that because of the potential risks (and the lack of consent), longitudinal studies should either invest in in-house processing facilities or await the consent of participants once they are adults. So they believe pediatric samples should not be shared or sent to researchers outside the facility. ${ }^{27}$ We think that the link between consent and risk is a flawed one. In much of the literature on biobanks, this link is made because informed consent is often seen as a way to transfer the responsibility of ill luck in outcomes from doctor (or researcher) to patient (or research 
participant). ${ }^{2,28}$ However, we think that the reasonable prevention of risks should precede consent, both in the case of adult and minor participants, because they or their parents should only be allowed to consent to relatively risklow research. Like Hoedemaekers et al, we assume that privacy risks can be adequately controlled by policy measures. ${ }^{6}$ Risks related to genetic privacy should be solved on two levels, first in society in general (through just institutions and genetic privacy laws) and second at a governance level, in appropriate data protection policies of biobanks, especially when samples are shared. In an empirical study about practices of 6 cohort studies involving children, representatives of these cohort studies emphasized strong data protection measures with coding and restricted access. Some studies also have established committees to advise on data handling issues such as requests by third parties. ${ }^{29}$

\section{Risk of Physical and Emotional Burden}

Literature on clinical trials often says research should only minimally burden children. ${ }^{30}$ Also, in the empirical research on opinions of lay people on the inclusion of children in biobank research, a major concern is it should not cause any burden on children. Specifically, the concern that the gathering of samples could cause children pain is a major impediment for the inclusion of children in research on tissue samples. A focus group study by Hens et al shows similar findings and stresses a preference that samples gathered in a diagnostics context be re-used. ${ }^{31}$ However, after physical discomfort, other types of burden are mentioned. For example, a focus group study by Kaufman et al shows that children might experience fear or that children might by overburdened by participation, especially because they had already a long list of activities. ${ }^{32}$ Goodenough et al interviewed minor participants of a large cohort study between the ages of 7 and 11 years and found that some would not enroll in the research again if they could choose again because it interfered too much with other activities. ${ }^{33}$ This is a risk that only occurs when samples are primarily gathered for research, and not in the case of the re-use of diagnostic collections, making this a major ethical difference in the two types of databases.

It is interesting to investigate why intuitively so much emphasis is put on the reduction of burden in the case of children, whereas the same type of burden seems to occur when adults are enrolled. This is related to children being more vulnerable than adults. This vulnerability has different aspects. First, it has to do with the limited autonomy of children. They are only enrolled in the research by means of the proxy consent of their parents. It may be perfectly all right to consent for oneself to research that is even more than minimally burdensome, but to do this for another, incompetent person is awkward. This is in sharp contrast to consenting for medical procedures for a child's benefit. Although the decision is awkward here as well, it is more readily made. A second, related aspect of the vulnerability is the lack of understanding of children. When it is not for their own benefit, children being hurt or frightened and not under- standing the reason why makes the procedure more problematic. Although we believe that children can be asked to contribute to research because of the principle of solidarity, it seems that, when such research involves an infringement of their specific vulnerability, this right to appeal is lessened or even non-existing.

The assessment of which kind of burden is acceptable is a hard one and may depend on specific circumstances and characteristics of a specific child. As aforementioned, the standard of "everyday risk" does not really apply here, because children who are often in contact with medical procedures may be more familiar with the procedures, but may be more vulnerable and thus exempt from yet another venepuncture. Also a 4-year-old child may be very upset at first by a venepuncture, but easily soothed and pleased when she receives a small sticker or child-friendly reward afterward. We believe that, because of the vulnerability aforementioned and the impossibility to create a burden threshold for all children, the precautionary principle should prevail here. This principle assumes that when there are good reasons on the basis of empirical evidence or causal hypothesis that damage could happen, adequate measures should be taken to prevent potential harmful outcomes. ${ }^{34}$ One important aspect would be that research protocols requiring blood or other samples for genetic research should try to re-use existing samples and data as much as possible. It is now sometimes the case that, because of strict consent procedures and the difficulty to gain consent for existing samples, new procedures are performed even when material from diagnostic collections was available. For example, large collections of blood-spot cards exist, which are gathered from newborns. As of yet, these are not used in many countries unless made anonymous because of the lack of consent for research and the administrative burden of trying to track parents or children for consent. Possibilities should be explored to use these cards in an ethical way. Also, procedures such as the use of oral fluid or local anesthetics should be explored when re-use of material is not possible. ${ }^{35}$

First, the ethics committee investigating a research proposal should make a assessment of the amount of burden research would pose on children and the alternatives. This committee should have a pediatrician as a member. Second, when a research proposal is considered acceptable, for each case, the researcher, the parent, and the child should decide on the desirability of a procedure. It is important that researchers are trained to assess reactions of the child in question, so that neither researcher nor parent would allow a procedure with would emotionally harm the child, solely for the purpose of the progress of science.

\section{Risk of Breaches of Values of Child}

The combination of children and stored sample collections, be it the reuse of diagnostic collection for research or the inception of new collections specifically for research, pose a difficulty that may not arise in clinical trials. On the one hand, samples can be stored in tissue sample collections for a longer 
period. Thus genetic research on them may spread over a longer period, and new types of research can be carried out on them. On the other hand, what is specific about minors is their lack of autonomy when they are small and their gradual growth toward autonomy and acquiring of certain values when they grow older. ${ }^{36}$ Typically, parents make many decisions for their small children, but would allow their children to gradually make more decisions for themselves as they grow older. Parents, as proxy decision makers, do not know the opinions of their children and thus make decisions on the basis of their own values and what they think is the best interest of their children. A related question is whether both parents should consent to the use of tissue from their child for genetic research. Because of the familiar nature of genetic information, this may be desirable. However, because many children are not living with both parents and consent from both parents may be not easy to obtain, this should probably be seen as a best practice rather than a strict rule. Thus it is possible that they would make decisions that their children would not make if they had to re-make the decision. This is, however, considered part of childhood and accepted, as long as children are allowed to develop their own values and opinions in the course of growing up, as also is shown in empirical literature. ${ }^{37}$ In the context of biobanks, this poses a specific risk. As research continues with time and takes new forms, it is possible that research done on samples for which parental consent was obtained when the child was 2 years old is contrary to the wishes of the participant who is now 16 years old. Especially when this research is non-reversible, this seems unacceptable, because children have the right to an open future and the right to make their own autonomous decisions when they are ready. ${ }^{38}$ For example, parents could have consented to a full genome scan of their child, the data of which are still circulating years later. Or they could have given broad consent to any research on samples, and the samples are at a given moment used for controversial research. Although we do not question the rights of parents to enroll their children in longitudinal biobank research, we also think it is important that the child, as a person who is gradually acquiring and exercising autonomy, is consulted and made aware of new research on her samples, especially when this research takes a great leap away from the original protocols. Thus we believe that parents should not be allowed to make any decisions that are too definite. Although to ask broad consent may be acceptable for adults, final decisions of this kind should be postponed until the child reaches the age of majority. This does not mean, however, that parents or older children should be asked to re-consent to each small change in research protocol. Ethics committees can decide whether a new type of research is sufficiently covered by the original consent or whether re-consent is needed. An objection may be that asking older minors to re-consent is too burdensome and may hamper research. However, technologies such as a website that would, given proper identification, allow an individual to review his or her consent could make the reconsent process more flexible. We agree that the feasibility of such re-consent also depends on the type of biobank and will be easier in the case of longitudinal cohort studies than in the case of the re-use of possibly anonymous samples from a diagnostics collection. In the latter case, the decision whether research can proceed without re-consent may depend on the evaluation of the ethics committee.

\section{Benefits}

We have described the restrictions that can and must be put on biobank research on children because of the potential risks. Restrictions can also be related to the types of research that is done. The most often quoted restrictions in this respect are related to either direct or group benefits or to such research only being able to be done on children when it cannot be done on adults.

For direct benefits, it is difficult to see how this criterion can applied to non-therapeutic genetic research on samples from children. Regular check-ups of children in case of longitudinal cohort studies may increase children's health when information is fed back to them, ${ }^{32}$ but direct benefit is never the primary aim of pediatric biobank research, and most of the children participating may never experience any health benefit from their participation. In this case, we think that the restriction "direct benefit" is too stringent in the case of pediatric biobanks. Also, there is a strong link between the amount of risk to which participants are exposed and the benefit requirement. In clinical trials, this is especially relevant. Children may be exposed to higher than minimal risk when the procedures are of potentially great benefit to them. Because we are talking about research on stored tissue samples and the risks are relatively lower than in clinical trials, the requirement of direct benefit is less important.

There are two other restrictions often quoted in the literature on medical research on children and in biobank guidelines that have their origin in the Declaration of Helsinki, which wanted to create an opening to allow research on subjects unable to consent. In its 2008 revision the declaration states: "These (ie, incompetent) individuals must not be included in a research study that has no likelihood of benefit for them unless it is intended to promote the health of the population represented by the potential subject, the research cannot instead be performed with competent persons, and the research entails only minimal risk and minimal burden." 39

The second restriction is a causal one and states that medical research can only be done on children when it cannot be done on adults. Several guidelines quote this restriction. ${ }^{14-}$

${ }^{16}$ We believe that this restriction is a straightforward and valid one to complement the restricted duty to solidarity that we advocate for children in genetic research.

The third restriction, of group benefits, is related to the post factum results of the research and states that research on minors should be for the benefit of children of the same age or with the same condition. Such provisions are quoted by the Council for International Organizations of Medical Science guideline, the World Health Organization, and the German Nationaler Etikrat. ${ }^{16,40,41}$ However, the requirement of 
group benefit is questioned by Holm. The argument behind the group benefit, he says, is that even when there is no direct benefit to the participant, he or she benefits indirectly through the group benefit. However, in the case of children, the membership is not stable when a disease only afflicts a particular age group, when a condition is rapidly progressive, when research is spread in a long period (such as is the case with cohort studies), or when the knowledge sought is the prevention of a certain disease. ${ }^{42}$ As we shall further argue, this is complicated when the research in question is genetic research, but we agree with Holm that there are certain problems when we try to limit research on children "intrinsically" by referring to either group benefits in the case of genetic biobank research. In the case of genetic research on stored tissue samples, it is hard to pin down exactly what the immediate benefits will be for children that have the condition. Imagine research that tries to find a relationship between autism and a gene mutation. Many parents of children with autism spectrum disorder will be happy to enroll their children for such research. Such research may indeed have beneficial consequences. An understanding of the relation between gene and condition may help parents of these children to cope with the condition, and this knowledge may relieve them from guilt. Thus it may benefit the children because it may ease the familial situation. Also, if newborns were screened for this specific gene mutation, it could prevent further harm by ensuring adequate follow-up and provide further assistance to families. However, some have argued that it is not always true that the earlier a disease is detected the better it is for the patient. ${ }^{43,44}$ And the discovery may also lead to the development of prenatal tests, which often lead to prevention through termination. Although the existence of such a test may be desired by certain parents, it is unclear how this fits the requirement of "benefitting children with the same condition." Thus the requirement of group benefit becomes more problematic with genetic research, because more steps are needed to reach the ultimate goal, medical benefit, and this goal may be unclear during the first, fundamental, steps of research. In the first instance, the goal of such research may be the good of science in general rather than the health of a specific group of children. Thus we doubt that the requirement of group benefits can easily be transferred from a clinical trial perspective to genetic biobank research. Therefore we would argue that the restriction "research cannot be done on adults" is a more straightforward one. We do not mean, however, that the consideration of benefit for other children is not important. It is the task of ethics committees reviewing protocols to always ask the questions, "Who benefits from this research, and what are the ultimate goals of research," with a risk assessment. Enrolling vulnerable subjects for the purpose of the progress of science only, without at least a possible positive end goal, is questionable. It could also erode trust in science with children who will once be competent adults. And although we acknowledge that clashes in values are ultimately unavoidable, they deserve consideration before research is undertaken.

\section{Conclusions and Recommendations}

Biobanks can appeal to the general public to participate in genetic research on the basis of the principle of solidarity, provided the aim of such research is to aid diagnosis and treatment of severe diseases or conditions. This counts for adults and for children. However, in the case of children, the solidarity principle is limited by certain considerations. Children are a vulnerable population because of their limited capabilities to make autonomous choices, their lack of understanding of the background and reason of certain procedures, and their right to an open future. Therefore, some limitations should be considered. These limitations are related to the requirement that such research should not pose more than minimal risk and to the type of research.

For the minimal risk standard, we first believe that the risks associated with genetic information should be solved on a policy and a societal level. Only when there is a sufficient guarantee that data are protected and cannot be accessed or misused by third parties should anyone (not only children) be asked to consent to biobank research. Second, we think that research should be as minimally burdensome as possible for children. Any physical or emotional harm should be avoided. This means that ethics committees should advise that all possibilities to re-use material gathered in a diagnostics environment are explored. When this is impossible, techniques such as buccal swabs or anesthetic creams with adequate rewards suitable for children and home visits should be used to make research as least intrusive as possible. Finally, it is the task of the researcher/research nurse, the parents, and the child together to check whether a specific procedure in a specific case is not too burdensome. Third, as biobank research may stretch in time and children grow in time toward more and more autonomy, their values should be respected. This means that parents should not be asked to give broad consent to each and any genetic research on their children's samples, but that re-consent of parent, child, or both should be evaluated when research takes a major leap forward from the original protocols.

Children and their parents have a responsibility to consider participation in genetic biobank research, which is also linked to some research just not being able to be done on adults. Therefore, we think this limitation, on the basis of the type of research, is a valid one. Another often quoted limitation, of benefit to other children, is more problematic for genetic research, because its final medical application is often uncertain at the time the research is done. However, we believe that any ethics committee should investigate the potential benefits of research on pediatric data and that at least the possibility of such benefit should exist.

We thank the anonymous reviewers for their valuable comments.

Submitted for publication Jun 29, 2010; last revision received Dec 6, 2010; accepted Dec 23, 2010. 
Reprint requests: Kristien Hens Katholieke Universiteit Leuven, Centre for Biomedical Ethics and Law, Kapucijnenvoer 35/3 Box 7001, 3000 Leuven, Belgium. E-mail: kristien.hens@med.kuleuven.be

\section{References}

1. Knoppers BM, Chadwick R. Human genetic research: emerging trends in ethics. Nat Rev Genet 2005;6:75-9.

2. Brekke OA, Sirnes T. Population biobanks: the ethical gravity of informed consent. BioSocieties 2006;1:385-98.

3. Beauchamp TL, Childress JF. Principles of biomedical ethics. New York: Oxford University Press; 1983.

4. Chadwick R, Berg K. Solidarity and equity: new ethical frameworks for genetic databases. Nat Rev Genetics 2001;2:318-21.

5. Hoedemaekers R, Gordijn B, Pijnenburg M. Solidarity and justice as guiding principles in genomic research. Bioethics 2007;21:342-50.

6. Hoedemaekers R, Gordijn B, Pijnenburg M. Does an appeal to the common good justify individual sacrifices for genomic research? Theor Med Bioeth 2006;27:415-31.

7. Bourgeois L. Solidarité. Marston Gate, UK: BiblioLife; 1902.

8. Burggraeve R. From self-development to solidarity. An ethical reading of human desire in its socio-political relevance according to Emmanuel Levinas. Leuven, Belguium: Peeters; 1985.

9. Harris J. Scientific research is a moral duty. J Med Ethics 2005;31:242-8.

10. Harris J, Holm S. Should we presume moral turpitude in our children?small children and consent to medical research. Theor Med Bioeth 2003; 24:121-9.

11. Hens K, Nys H, Cassiman JJ, Dierickx K. Biological sample collections from minors for genetic research: a systematic review of guidelines and position papers. Eur J Hum Genet 2009;17:979-90.

12. Hens K, Nys H, Cassiman JJ, Dierickx K. Genetic research on stored tissue samples from minors: a systematic review of the ethical literature. Am J Med Genet A 2009;149A:2346-58.

13. Wendler D, Belsky L, Thompson KM, Emanuel EJ. Quantifying the federal minimal risk standard: implications for pediatric research without a prospect of direct benefit. JAMA 2005;294:826-32.

14. Irish Council for Bioethics. Human biological material: recommendations for collection, use and storage in research. Dublin, Ireland: Irish Council for Bioethics; 2005.

15. Nuffield Council on Bioethics. Human tissue, ethical and legal issues. London, UK: Nuffield Council on Bioethics; 1995.

16. Council for International Organizations of Medical Science (CIOMS). International ethical guidelines for biomedical research involving human subjects. Geneva; 2002.

17. Leikin S. Epidemiological research with children. In: Coughlin SS, Beauchamp TL, eds. Ethics and epidemiology. New York \& Oxford: Oxford University Press; 1996. p. 199-218.

18. Eriksson S, Helgesson G. Potential harms, anonymization, and the right to withdraw consent to biobank research. Eur J Hum Genet 2005;13:1071-6.

19. Boenink $M$, van der Burg S. Informed decision making about predictive DNA tests: arguments for more public visibility of personal deliberations about the good life. Med Health Care Philos 2010;13:127-38.

20. van Diest PJ. No consent should be needed for using leftover body material for scientific purposes. BMJ 2002;325:648-51.

21. Roche PA, Annas GJ. Protecting genetic privacy. Nat Rev Genetics 2001; 2:392-6.

22. Annas GJ. Privacy rules for DNA databanks. Protecting coded "future diaries.". JAMA 1993;270:2346-50.
23. Beck U. Risk society. Towards a new modernity. London: Sage Publications; 1992.

24. Van Camp N, Dierickx K. The expansion of forensic DNA databases and police sampling powers in the post-9/11 era: ethical considerations on genetic privacy. Ethical Perspectives 2007;14:237-68.

25. Lin Z, Altman RB, Owen AB. Confidentiality in genome research. Science 2006;313:441-2.

26. Baumann TK. Proxy consent and a national DNA databank: an unethical and discriminatory combination. Iowa Law Rev 2001;86: 667-701.

27. Gurwitz D, Fortier I, Lunshof JE, Knoppers BM. Research ethics. Children and population biobanks. Science 2009;325:818-9.

28. Dickenson D. Risk and luck in medical ethics. Oxford: Blackwell; 2003.

29. Ries NM, Legrandeur J, Caulfield T. Handling ethical, legal and social issues in birth cohort studies involving genetic research: responses from studies in six countries. BMC Med Ethics 2010;11:4.

30. Pinxten W, Dierickx K, Nys H. Ethical principles and legal requirements for pediatric research in the EU: an analysis of the European normative and legal framework surrounding pediatric clinical trials. Eur J Pediatr 2009;168:1225-34.

31. Hens K, Nys H, Cassiman JJ, Dierickx K. The storage and use of biological tissue samples from minors for research: a focus group study. Public Health Genomics. In press 2010.

32. Kaufman D, Geller G, LeRoy L, Murphy J, Scott J, Hudson K. Ethical implications of including children in a large biobank for geneticepidemiologic research: a qualitative study of public opinion. Am J Med Genetics. Part C Semin Med Genetics 2008;148C:31-9.

33. Goodenough T, Williamson E, Kent J, Ashcroft R. "What did you think about that?" Researching children's perceptions of participation in a longitudinal genetic epidemiological study. Child Soc 2003;17: 113-225.

34. Andorno R. The precautionary principle: a new legal standard for a technological age. J Intern Biotechnol Law 2004;1:11-8.

35. Bartington SE, Peckam C, Brown D, Joshi H, Deateux C. Feasibility of collecting oral fluid samples in the home setting to determine seroprevalence of infections in a large-scale cohort of preschool-aged children. Epidemiol Infect 2009;137:211-8.

36. Matthews GB. The philosophy of childhood. Cambridge and London: Harvard University Press; 1994.

37. Hens K, Nys H, Cassiman JJ, Dierickx K. The storage and use of biological tissue samples from minors for research: a focus group study. Public Health Genomics. In press 2010.

38. Feinberg J. The child's right to an open future. In: Aiken W, LaFollette H, eds. Whose child? Children's rights, parental authority and state power. Totowa, NJ: Rowman and Littlefield; 1980. p. 124-53.

39. World Medical Association (WMA). Declaration of Helsinki. Ethical principles for medical research involving human subjects. 2008.

40. World Health Organization. Genetic databases. Assessing the benefits and the impacts on human and patient rights. 2003.

41. Nationaler Ethikrat. Biobanks for research. Berlin, Germany: Nationaler Ethikrat; 2004.

42. Holm S. Informed consent and the bio-banking of material from children. Genomics Soc Policy 2005;1:16-26.

43. Malm HM. Medical screening and the value of early detection. When unwarranted faith leads to unethical recommendations. Hastings Center Rep 1999;29:26-37.

44. Dierickx K. The ethics of population screening. In: Ten Have R, Chadwick R, eds. Handbook for health care ethics (forthcoming). London: Sage Publications; 2010. 